Artigo Original

\title{
Excesso de adiposidade corporal em adolescentes: associação com fatores sociodemográficos e aptidão física
}

\author{
Diego Augusto Santos Silva ${ }^{1}$ \\ Thales Boaventura Rachid Nascimento ${ }^{2}$ \\ Adelson Fernandes da Silva ${ }^{3}$ \\ Maria Fátima Glaner ${ }^{4}$ \\ ${ }^{1}$ Universidade Federal de Santa Catarina, Programa de Pós-Graduação em Educação Física, Núcleo \\ de Pesquisa em Cineantropometria e Desempenho Humano, Florianópolis, SC, Brasil. \\ ${ }^{2}$ Bolsista da Coordenação de Aperfeiçoamento de Pessoal de Nível Superior (CAPES) Universidade \\ Católica de Brasília, Programa de Pós Graduação em Educação Física, Brasília, DF, Brasil. \\ ${ }^{3}$ Universidade Estadual de Montes Claros, Januária, Minas Gerais, Brasil. \\ ${ }^{4}$ Universidade Estadual de Londrina, Centro de Educação Física e Esporte, Departamento de \\ Educação Física, Londrina, PR, Brasil.
}

\begin{abstract}
Resumo: Objetivou-se verificar a prevalência e os fatores sociodemográficos e de aptidão física associados à adiposidade corporal elevada (ACE) em adolescentes de Januária-MG, Brasil. A amostra foi de 266 rapazes e 361 moças $(15,47 \pm 1,06$ anos). A adiposidade foi estimada pelo somatório das dobras cutâneas tricipital e subescapular. As variáveis independentes foram: zona de domicílio (rural e urbana); idade (14-15 e 16-17); nível econômico (alto, médio e baixo); aptidão aeróbia, flexibilidade e resistência muscular (adequada e inadequada). Nos rapazes, 13,91\% apresentaram ACE, com maior somatório de dobras cutâneas sendo observado nos residentes da zona urbana $(p<0,05)$. Nas moças, $33,24 \%$ apresentara ACE, sendo que aquelas com 16-17 anos e de nível econômico mais baixo apresentaram maiores valores de somatório de dobras cutâneas $(p<0,05)$. A ACE associou-se, apenas nos rapazes, a zona urbana e à resistência abdominal inadequada $(p<0,05)$. Assim, em comparação as moças, os rapazes parecem ser mais vulneráveis aos fatores ambientais.
\end{abstract}

Palavras-chave: Obesidade. Sobrepeso. Pregas cutâneas. Estudos transversais. Saúde do Adolescente.

High body fat among adolescents: association with sociodemographics and physical fitness factors

\begin{abstract}
The objective was to verify the prevalence and factors associated with high body fat in

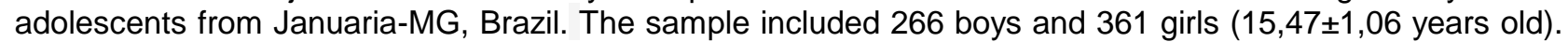
Adiposity was estimated by the sum of triceps and subscapular skinfold thickness. The independent variables were: area of residence (urban and rural); age (14-15 and 16-17 years old); house income (high, medium and low); aerobic fitness, flexibility and muscular resistance (adequate and inadequate). Among boys, $13,91 \%$ exhibited adiposity excess, with higher $(p<0.05)$ skinfold thicknesses sum been observed among boys with low house income and urban residence. Among girls, 33,24\% exhibited adiposity excess, with higher $(p<0.05)$ skinfold thicknesses sum been observed among girls with 16-17 years old and low house income. Adiposity excess was associates, only in boys, with urban residence and inadequate muscular resistance. Thus, boys seem to be more susceptible to environmental factors than girls.
\end{abstract}

Keywords: Obesity. Overweight. Skinfold Thickness. Cross-Sectional Studies. Adolescent Health.

\section{Introdução}

A obesidade, já na adolescência, está associada ao desenvolvimento de fatores de risco para doenças cardiovasculares, tais como hipertensão arterial, dislipidemias, diabetes tipo II, síndrome metabólica e apneia do sono, bem como a desordens ortopédicas (PEEBLES, 2008).

A gênese da obesidade é multifatorial, sendo essa resultante da interação de fatores genéticos, hormonais, psicológicos (PEEBLES, 2008), alimentares (GÓMEZ-MARTÍNEZ et al., 2012; DUNCAN et al., 2011). Porém, outros fatores, como nível econômico (DUQUIA et al., 2008; LIU et al., 2008; VICENTE-RODRÍGUEZ et al., 2008) e zona de domicilio (GLANER, 2005a; PELEGRINI et al., 2010; TASSITANO et al., 2009; LI et al., 2008), estão relacionados ao excesso de adiposidade na adolescência.

Em relação aos fatores não modificáveis, pode-se citar a idade. Em adolescentes, ainda há controvérsias sobre qual grupo etário apresenta maiores chances de adiposidade corporal elevada. Pesquisadores observaram que em jovens com idade superior aos 13 anos essas chances são menores ( $\underline{X U}$ et al., 2008; VICENTERODRÍGUEZ et al., 2008), ao passo que outros evidenciam maior chance de excesso de peso na faixa etária de 7 a 10 anos (DUNCAN et al., 
2011). Em contrapartida, há estudos que não evidenciaram associação da idade com a adiposidade corporal excessiva (SILVA et al., 2011; PELEGRINI et al., 2010; TASSITANO et al., 2009).

No que se refere aos fatores modificáveis, a literatura também é controversa. Estudos demonstraram que a adiposidade corporal elevada se apresenta associada, a níveis econômicos mais altos (DUQUIA et al., 2008) ou mais baixos (LIU et al., 2008; VICENTERODRÍGUEZ et al., 2008), dependendo do local de investigação. Outra variável, que vem sendo estudada como possível fator associado à obesidade é a zona de domicílio. Pesquisadores relataram maior prevalência (GLANER, 2005a) e risco (PELEGRINI et al., 2010; TASSITANO et al., 2009; 느 et al., 2008) de adiposidade corporal elevada entre os adolescentes que residem em locais urbanizados, quando comparado aqueles das zonas rurais.

A aptidão física é tida como um dos mais importantes indicadores de saúde, haja vista sua relação inversa com diversos fatores de risco cardiovascular (ORTEGA et al., 2008). Dos vários componentes da aptidão física, tem-se a aptidão aeróbia, a qual apresenta relação inversa com os indicadores de adiposidade corporal (DUMITH et al., 2010; MOLINER-URDIALES et al., 2011; SILVA et al. 2011). Apesar disso, a relação entre adiposidade corporal e os demais componentes da aptidão física ainda é pouco explorada.

A técnica de dobras cutâneas apresenta boa estimativa da gordura corporal em adolescentes (GLANER, 2005b; FREEDMAN et al., 2007). Ademais, em comparação a métodos mais precisos para estimar a composição corporal, tal como a ressonância magnética e a pletismografia, a técnica de dobras cutâneas apresenta baixo custo.

Adolescentes obesos apresentam maior risco para a obesidade severa ao atingir a fase adulta (THE et al., 2010). Portanto, identificar a prevalência e os fatores associados à obesidade é fundamental para a elaboração e direcionamento de medidas preventivas. Assim, o presente estudo teve como objetivo verificar a prevalência e os fatores sociodemográficos e de aptidão física associados à adiposidade corporal elevada em adolescentes.

\section{Métodos}

\section{Local do estudo}

A coleta de dados do presente estudo foi realizada no município de Januária-MG, situada na região do Médio São Francisco do estado brasileiro de Minas Gerais, no ano de 2009. O estudo foi submetido e aprovado pelo Comitê de Ética das Faculdades Unidas do Norte de Minas (Parecer 0129/09).

O município de Januária-MG é constituído por 65.463 habitantes (IBGE, 2010). O Índice de Desenvolvimento Humano (IDH) é de 0,699 e classifica 0 município com médio-baixo desenvolvimento humano (NU, 2000).

\section{População e Amostra}

Matriculados no ensino fundamental e médio, o município apresentava um total de 15.464 estudantes, sendo $95,5 \%$ oriundos das escolas públicas. Destes, 11.215 e 3.553 cursavam o ensino fundamental e médio, respectivamente (IBGE, 2010).

O processo de amostragem foi estratificado em dois estágios: 1) escolas públicas de ensino fundamental e médio e; 2) conglomerado de turmas. No primeiro estágio, foram consideras, apenas, as escolas que tinham ensino fundamental e médio $(n=08)$, pois eram as maiores do município e concentravam o maior número de alunos. Sendo assim, foram excluídas duas escolas que tinham somente ensino fundamental (uma rural e outra urbana) e uma de ensino médio (urbana). Das oito escolas selecionadas, cinco eram localizadas na zona rural e três na zona urbana. Tendo em vista a maior quantidade de alunos na zona urbana, optou-se por selecionar mais escolas na zona rural, a fim de evitar que a amostra ficasse desproporcional. No segundo estágio, todos os adolescentes entre 14 e 17 anos, matriculados nas escolas selecionadas, foram convidados a participar do estudo.

Este estudo faz parte de uma pesquisa mais ampla intitulada "Análise da atividade física e aptidão física relacionada à saúde em escolas rurais e urbanas". Foram calculados vários tamanhos de amostra com diferentes desfechos em saúde. Para a presente análise adotou-se prevalência desconhecida para o desfecho (igual a $50 \%$ ), erro tolerável de cinco pontos percentuais, nível de confiança de $95 \%$, efeito de delineamento de 1,5 , acrescentando $15 \%$ para possíveis perdas e recusas. Considerando que no município de Januária, 4.495 escolares estavam matriculados no ensino fundamental e médio, estimou-se uma amostra de 611 adolescentes. Como foram convidados a participar do estudo todos os adolescentes presentes em sala de aula no dia da coleta de dados, a amostra final foi composta por 627 adolescentes, sendo 328 da zona rural e 299 da zona urbana. 
Os critérios de exclusão adotados foram: (a) faixa etária fora da determinada para a pesquisa; (b) não apresentar o Termo de Consentimento Livre e Esclarecido - TCLE, assinado pelos responsáveis. Considerou-se recusa o adolescente não querer participar da pesquisa.

\section{Procedimentos para coleta de dados}

A coleta de dados foi realizada por professores e alunos do curso de Educação Física, os quais foram previamente treinados. As avaliações antropométricas (massa corporal, estatura e espessura de dobras cutâneas) foram realizadas por um único avaliador (aluno de mestrado em Educação Física) com experiência nas mensurações pré-determinadas. Os demais integrantes da equipe de coleta de dados obtiveram informações sociodemográficas (sexo, idade, nível econômico e área de domicílio) por meio de questionários e de aptidão física (flexibilidade, resistência muscular abdominal e aptidão aeróbia).

As informações sociodemográficas foram coletadas por meio de um questionário autoadministrado em sala de aula. A variável idade foi coletada de forma contínua e posteriormente categorizada em 14-15 anos e 1617 anos. A área de domicílio foi dividida em zona rural e urbana. Os alunos que estudavam na zona urbana e residiam na zona rural foram considerados como pertencentes à zona rural. $\mathrm{O}$ nível econômico foi identificado pelo questionário da Associação Brasileira de Empresas de Pesquisa (2008) que classifica a população brasileira em cinco classes econômicas, por ordem decrescente de poder de compra ("A", "B", "C", " $D$ " e "E"). No presente estudo, agruparam-se as categorias "A" + "B", considerado nível econômico alto; "C", nível médio e "D" + "E" nível econômico baixo.

As variáveis antropométricas (massa corporal, estatura, espessura de dobras cutâneas tricipital e subescapular) foram mensuradas seguindo procedimentos padronizados (PETROSKI, 2009). A massa corporal $(\mathrm{kg})$ foi obtida por meio de uma balança digital com escala de medida de 100 gramas. Para estatura $(\mathrm{cm})$ utilizou- se um estadiômetro, com escala de $0,1 \mathrm{~cm}$ Para a mensuração da espessura das dobras cutâneas $(\mathrm{mm})$ foi utilizado um compasso, com escala de $0,1 \mathrm{~mm}$. A adiposidade corporal foi estimada por meio do somatório das dobras cutâneas tricipital e subescapular $(\Sigma 2 \mathrm{DC})$, e classificada em: baixa $(\Sigma 2 \mathrm{DC}<13 \mathrm{~mm}$ ou $<15 \mathrm{~mm})$, normal $\left(\sum 2 \mathrm{DC}\right.$ entre 13 e $22 \mathrm{~mm}$ ou entre 15 e $27 \mathrm{~mm}$ ) e elevada $(\Sigma 2 \mathrm{DC}>22 \mathrm{~mm}$ ou $>27 \mathrm{~mm}$ ), para rapazes $\mathrm{e}$ moças, respectivamente (LOHMAN, 1992). Para os objetivos do presente estudo agrupou-se a categoria baixa com a normal.

Para a obtenção dos resultados referentes aos testes de aptidão física empregou-se a bateria de teste Physical Best (AAHPERD, 1988), a qual foi realizada nas quadras das escolas. A flexibilidade foi medida por meio do teste de sentar e alcançar, e a força/resistência muscular, da parte inferior do tronco, por meio do teste de repetições abdominais durante $1 \mathrm{~min}$. A aptidão aeróbia foi estimada pelo teste de correr/caminhar $1600 \mathrm{~m}$. Para tanto, foi demarcado, previamente, um espaço em que os adolescentes teriam que percorrer a referida distância no menor tempo possível. Os resultados dos testes físicos foram classificados em adequados ou inadequados para a saúde (AAHPERD, 1988).

\section{Análise estatística}

$\mathrm{Na}$ análise descritiva das variáveis foram utilizadas médias, desvios padrão e distribuição de frequências. A comparação entre os sexos foi realizada por meio do teste " $\mathrm{t}$ " de Student para amostras independentes. O teste "t" de Student para amostras independentes também foi empregado para comparar o somatório de dobras cutâneas de acordo com as categorias de idade, variáveis sociodemográficas e de aptidão física, estratificado por sexo. A Análise de Variância "one way" com o teste post hoc de Tukey foi empregada para comparar o somatório de dobras cutâneas de acordo com as categorias de nível econômico, estratificado por sexo.

Ao verificar que a variável dependente (excesso de adiposidade corporal) apresentou prevalência elevada, utilizou-se a regressão de Poisson com ajuste robusto para variância, com o propósito de examinar as associações deste desfecho com os indicadores sociodemográficos (idade, nível econômico e área de domicílio) e de aptidão física (flexibilidade, resistência abdominal e aptidão aeróbia), estimando-se razões de prevalência e os intervalos de confiança de $95 \%$ (IC95\%). Todas as variáveis foram introduzidas no modelo ajustado independente do valor de " $p$ " a análise bruta. No entanto, a análise foi realizada de forma hierarquizada, seguindo as recomendações da literatura (VICTORA et al., 1997). A análise foi dividida em dois blocos: 1) variáveis sociodemográficas (Distal); 2) variáveis de aptidão física (Proximal). Permaneceram no modelo as variáveis com valor de $p<0,20$ (MALDONADO; GREENLAND, 1992). O nível de significância foi estabelecido em 5\% (IC95\%).

\section{Resultados}

O estudo apresentou taxa de resposta de $80,5 \%$. Assim, a amostra foi composta de 627 
adolescentes com 15,47 (DP $=1,06)$ anos de idade, sendo 266 rapazes (56,02\% rurais) e 361 moças $(49,58 \%$ rurais).

$\mathrm{Na}$ tabela 1 são apresentados os valores descritivos e a comparação da idade, variáveis antropométricas e de aptidão física entre os sexos. Os rapazes, em relação às moças, apresentaram maiores valores para massa corporal, estatura e melhor desempenho nos testes de resistência abdominal e aptidão aeróbia $(p<0,05)$. As moças apresentaram maiores valores para a idade e somatório de dobras cutâneas $(p<0,05)$. Para a flexibilidade não houve diferenças entre os sexos $(p>0,05)$.

Tabela 1. Comparação dos valores médios $(\bar{X})$ e desvio padrão (dp) da idade, variáveis antropométricas e de aptidão física entre os sexos. Januária-MG, Brasil.

\begin{tabular}{lcc}
\hline Variáveis & Rapazes & Moças \\
& $\bar{X}(\mathrm{dp})$ & $\bar{X}(\mathrm{dp})$ \\
\hline Idade (anos) & $15,38(1,09)$ & $15,56(1,05)^{\star}$ \\
Massa corporal (kg) & $55,94(10,68)$ & $51,64(7,47)^{\star}$ \\
Estatura (cm) & $168,08(8,97)$ & $160,37(6,29)^{\star}$ \\
2DC (mm) & $16,52(9,11)$ & $25,01(9,59)^{\star}$ \\
Flexibilidade (cm) & $26,25(7,52)$ & $25,65(7,97)$ \\
Resistência Abdominal (rep) & $25,95(7,63)$ & $17,82(7,55)^{\star}$ \\
Aptidão Aeróbia (min) & $9: 10(2: 40)$ & $12: 26(3: 14)^{\star}$ \\
\hline
\end{tabular}

$\sum 2 \mathrm{DC}=$ somatório das dobras cutâneas do tríceps e subescapular.

* Diferença $(p<0,05)$ em relação aos rapazes.

Tabela 2. Comparação dos valores médios $(\bar{X})$ e desvio padrão (dp) do somatório de dobras cutâneas de acordo com a idade, variáveis sociodemográficas e de aptidão física nos rapazes e nas moças. Januária-MG, Brasil.

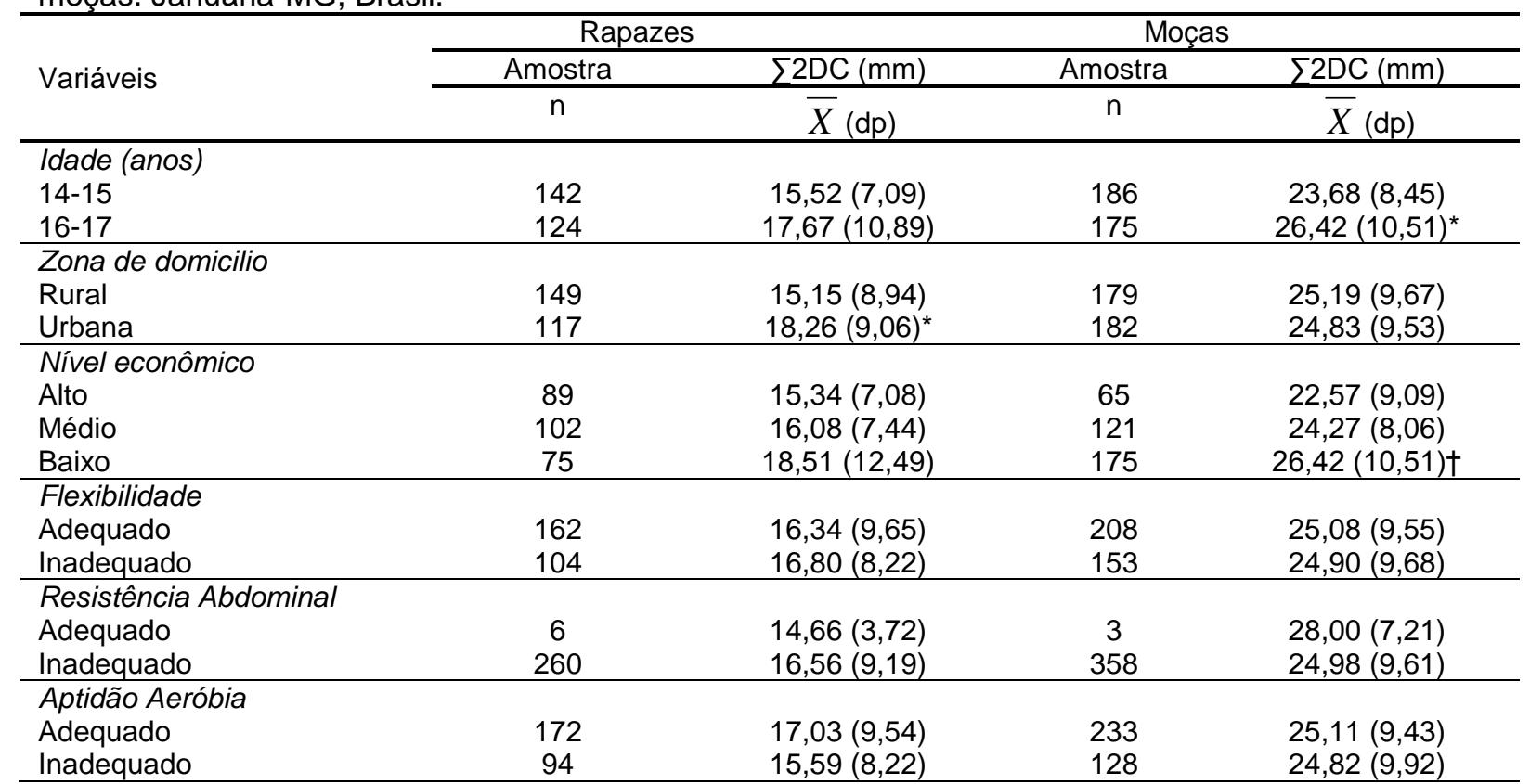

$\Sigma \overline{2 \mathrm{DC}}=$ somatório das dobras cutâneas do tríceps e subescapular; ${ }^{*} p<0,05$ (Teste "t" de Student para amostras independentes - comparação realizada para cada sexo separadamente); $\uparrow p<0,05$ - valor mais elevado do que o nível econômico Alto (Análise de variância "one way" com o teste post hoc de Tukey).

$\mathrm{Na}$ tabela 2 pode-se observar que rapazes residentes na zona urbana apresentaram maiores valores do somatório de dobras cutâneas do que os rapazes da zona rural $(p<0,05)$. Em relação às moças, as de 16-17 anos e de nível econômico baixo, apresentaram maiores valores de somatório de dobras cutâneas do que as de 14-15 anos e de nível econômico alto, respectivamente.
A maioria dos rapazes estava na faixa etária de 14-15 anos, residia na zona rural e pertencia ao nível econômico médio. A prevalência de adiposidade corporal elevada nos rapazes foi de $13,91 \%$. Ademais, a prevalência de adiposidade corporal elevada de acordo com as variáveis independentes está na Tabela 3. 
Tabela 3. Distribuição da amostra e prevalência de adiposidade corporal elevada e intervalos de confiança (IC) de acordo com as variáveis sociodemográficas e da aptidão física em adolescentes. Januária-MG, Brasil.

\begin{tabular}{|c|c|c|c|c|}
\hline \multirow{3}{*}{ Variáveis } & \multicolumn{2}{|c|}{ Rapazes } & \multicolumn{2}{|c|}{ Moças } \\
\hline & Amostra & Adiposidade elevada & Amostra & Adiposidade elevada \\
\hline & $\mathrm{n}$ & $\%(\mathrm{IC} 95 \%)$ & $\mathrm{n}$ & $\%(\mathrm{IC} 95 \%)$ \\
\hline Total & 266 & $13,91(9,72-18,09)$ & 361 & $33,24(28,35-38,12)$ \\
\hline \multicolumn{5}{|l|}{ Idade (anos) } \\
\hline $14-15$ & 142 & $11,27(6,00-16,53)$ & 186 & $28,49(21,94-35,04)$ \\
\hline $16-17$ & 124 & $16,93(10,24-23,62)$ & 175 & $38,28(31,01-45,58)$ \\
\hline \multicolumn{5}{|l|}{ Zona de domicilio } \\
\hline Rural & 149 & $8,72(4,14-13,30)$ & 179 & $33,51(26,53-40,50)$ \\
\hline Urbana & 117 & $20,51(13,08-27,93)$ & 182 & $32,96(26,07-39,86)$ \\
\hline \multicolumn{5}{|l|}{ Nível econômico } \\
\hline Alto & 89 & $13,48(6,24-20,71)$ & 65 & $23,07(12,55-33,59)$ \\
\hline Médio & 102 & $12,74(6,16-19,32)$ & 121 & $31,40(23,01-39,79)$ \\
\hline Baixo & 75 & $16,00(7,50-24,49)$ & 175 & $38,28(31,01-45,05)$ \\
\hline \multicolumn{5}{|l|}{ Flexibilidade } \\
\hline Adequado & 162 & $12,96(7,73-18,19)$ & 208 & $33,17(26,72-39,62)$ \\
\hline Inadequado & 104 & $15,38(8,33-22,43)$ & 153 & $33,33(25,77-40,88)$ \\
\hline \multicolumn{5}{|l|}{ Resistência Abdominal } \\
\hline Adequado & 6 & $0,00(0,00-0,00)$ & 3 & $33,33(10,08-76,75)$ \\
\hline Inadequado & 260 & $14,23(9,95-18,50)$ & 358 & $33,24(28,33-38,14)$ \\
\hline \multicolumn{5}{|l|}{ Aptidão Aeróbia } \\
\hline Adequado & 172 & $13,95(8,72-19,18)$ & 233 & $34,33(28,19-40,47)$ \\
\hline Inadequado & 94 & $13,82(6,72-20,93)$ & 128 & $31,25(23,11-39,38)$ \\
\hline
\end{tabular}

A maior parte das moças estava na faixa etária de 14-15 anos, residia na zona urbana e pertencia ao nível econômico baixo. A prevalência de adiposidade corporal elevada nas moças foi de $33,24 \%$. A prevalência de adiposidade corporal elevada de acordo com as variáveis independentes está na Tabela 3.

Tabela 4. Razões de prevalência (RP) e intervalos de confiança (IC) estimados por meio da Regressão de Poisson entre adiposidade corporal elevada e variáveis sociodemográficas e de aptidão física - nos rapazes. Januária-MG, Brasil.

\begin{tabular}{lcc}
\hline Variáveis & $\begin{array}{c}\text { Análise bruta } \\
\mathrm{RP}(\mathrm{IC} 95 \%)\end{array}$ & $\begin{array}{c}\text { Análise ajustada } \\
\mathrm{RP}(\mathrm{IC} 95 \%)\end{array}$ \\
\hline $\begin{array}{l}\text { Idade } \\
14-15\end{array}$ & 1 & 1 \\
$16-17$ & $1,05(0,97-1,13)$ & $1,07(0,95-1,19)$ \\
\hline $\begin{array}{l}\text { Zona de domicilio } \\
\text { Rural }\end{array}$ & 1 & 1 \\
Urbana & $1,11(1,03-1,19)^{*}$ & $1,11(1,03-1,19)^{*}$ \\
\hline Nível econômico & $0,98(0,89-1,07)$ & $1,01(0,87-1,17)$ \\
Alto & $0,97(0,88-1,06)$ & $0,98(0,87-1,09)$ \\
Médio & 1 & 1 \\
Baixo & 1 & 1 \\
\hline Flexibilidade & $1,02(0,94-1,10)$ & $1,02(0,94-1,09)$ \\
Adequado & & 1 \\
Inadequado & 1 & $1,22(1,14-1,30)^{*}$ \\
\hline Resistência Abdominal & $1,14(1,10-1,18)^{*}$ & 1 \\
$\begin{array}{l}\text { Adequado } \\
\text { Inadequado }\end{array}$ & 1 & $0,97(0,90-1,04)$ \\
\hline $\begin{array}{l}\text { Aptidão Aeróbia } \\
\text { Adequado }\end{array}$ Inadequado & $1,00(0,92-1,07)$ & \\
\hline${ }^{*}$ p<0,01 & &
\end{tabular}

Em relação aos rapazes, tanto na análise bruta quanto na ajustada, os grupos com maiores prevalências de excesso de adiposidade corporal foram os adolescentes que residiam na zona urbana e aqueles com resistência abdominal inadequada $(p<0,05)$ (Tabela 4).
Em relação às moças, na análise bruta, os grupos com maiores prevalências de adiposidade corporal elevada foram as adolescentes na faixa etária entre 16-17 anos e de nível econômico mais baixo. Após o ajuste, nenhuma variável manteve-se associada ao desfecho (Tabela 5). 
Tabela 5. Razões de prevalência (RP) e intervalos de confiança (IC) estimados por meio da Regressão de Poisson entre adiposidade corporal elevada e variáveis sociodemográficas e de aptidão física nas moças. Januária-MG, Brasil.

\begin{tabular}{|c|c|c|}
\hline Variáveis & $\begin{array}{l}\text { Análise bruta } \\
\text { RP (IC95\%) }\end{array}$ & $\begin{array}{c}\text { Análise ajustada } \\
\text { RP (IC95\%) }\end{array}$ \\
\hline $\begin{array}{l}\text { ldade } \\
14-15 \\
16-17\end{array}$ & $\begin{array}{c}1 \\
1,07(1,01-1,16)^{*}\end{array}$ & $\begin{array}{c}1 \\
1.05(0.96-1.14)\end{array}$ \\
\hline $\begin{array}{l}\text { Zona de domicilio } \\
\text { Rural } \\
\text { Urbana }\end{array}$ & $\begin{array}{c}1 \\
0,99(0,91-1,07)\end{array}$ & $\begin{array}{c}1 \\
0,99(0,92-1,07)\end{array}$ \\
\hline $\begin{array}{l}\text { Nível econômico } \\
\text { Alto } \\
\text { Médio } \\
\text { Baixo }\end{array}$ & $\begin{array}{c}0,89(0,80-0,98)^{*} \\
0,95(0,87-1,03) \\
1\end{array}$ & $\begin{array}{c}0,93(0,84-1,03) \\
0,95(0,87-1,03) \\
1\end{array}$ \\
\hline $\begin{array}{l}\text { Flexibilidade } \\
\text { Adequado } \\
\text { Inadequado }\end{array}$ & $\begin{array}{c}1 \\
1,01(0,93-1,07)\end{array}$ & $\begin{array}{c}1 \\
1,00(0,93-1,07)\end{array}$ \\
\hline $\begin{array}{l}\text { Resistência Abdominal } \\
\text { Adequado } \\
\text { Inadequado }\end{array}$ & $\begin{array}{c}1 \\
1,00(0,66-1,49)\end{array}$ & $\begin{array}{c}1 \\
0,99(0,67-1,46) \\
\end{array}$ \\
\hline $\begin{array}{l}\text { Aptidão Aeróbia } \\
\text { Adequado } \\
\text { Inadequado }\end{array}$ & $\begin{array}{c}1 \\
0,97(0,90-1,05)\end{array}$ & $\begin{array}{c}1 \\
0,97(0,90-1,05)\end{array}$ \\
\hline
\end{tabular}

\section{Discussão}

O principal achado do presente estudo foi que aproximadamente $25 \%$ dos investigados apresentaram excesso de adiposidade corporal, sendo essa prevalência maior nas moças em comparação aos rapazes. Além disso, pode-se observar que dentre os fatores sociodemográficos e de aptidão física analisados, o domicílio urbano e a resistência abdominal inadequada estiveram associados à adiposidade corporal elevada nos rapazes. Nas moças, nenhuma variável investigada associou-se ao desfecho.

O melhor desempenho no teste de resistência abdominal e aptidão aeróbia, os maiores valores de massa corporal e estatura, e os menores indicadores de gordura corporal, do sexo masculino em comparação ao feminino, estão em conformidade com outros estudos (MOLINER$\underline{\text { URDIALES }}$ et al., 2011; $\underline{\text { DUMITH }}$ et al., 2010; DELAGRANNA et al., 2010). Para a flexibilidade, os resultados semelhantes entre os sexos convergem (DELAGRANNA et al., 2010) e divergem (DUMITH et al., 2010). Essas diferenças para o desempenho físico e variáveis antropométricas são provenientes, em grande parte, dos fatores hormonais, os quais durante a adolescência exercem maior influência no aumento de força e massa muscular nos rapazes em comparação as moças, as quais tendem a acumular maior volume de gordura subcutânea (MALINA, 1991).

A prevalência de adiposidade corporal elevada foi de $13,91 \%$ nos rapazes e de $33,24 \%$ nas moças, o que é superior ( $8 \%$ nos rapazes e $3,1 \%$ nas moças) ao observado em escolares da região sul (SILVA et al., 2011) e inferior (25,4\% nos rapazes e $41,2 \%$ nas moças) aos da região nordeste do Brasil (SILVA et al., 2007). Essas diferenças entre os estudos podem ser provenientes das peculiaridades no estilo de vida de cada localidade e ao próprio erro de amostragem.

Residir na área urbana está associado, em adolescentes de ambos os sexos, a maior ocorrência de sobrepeso e obesidade (PELEGRINI et al., 2010; 느 et al., 2008; GLANER, 2005a), sendo que morar na zona rural confere uma menor chance de ter excesso de peso ( $\underline{\mathrm{XU}}$ et al., 2008). No presente estudo, residir na área urbana conferiu, apenas aos rapazes, maior risco $[R P=1,11 ; \quad I C 95 \%: 1,03-1,19]$ de adiposidade corporal elevada, mesmo após ajuste para outras variáveis. Esse resultado se assemelha ao observado em adolescentes de Pernambuco, em que uma maior chance para o sobrepeso e obesidade, verificado por meio do IMC, foi observada nos rapazes domiciliados na zona urbana, mas não nas moças (TASSITANO et al., 2009). 
Em comparação aos adolescentes residentes na zona urbana, os da zona rural são mais fisicamente ativos (GORDIA et al., 2010), possivelmente em razão desses ajudarem seus pais nas atividades laborais. No meio rural, as moças se dedicam mais as atividades domésticas, enquanto os rapazes às atividades do campo. Já na zona urbana, somente a atuação das moças, no trabalho doméstico, é observada. Assim, é possível que a diferença no nível de atividade física entre rurais e urbanos seja maior entre os rapazes. Por consequência, apenas nesse grupo, é possível observar associação entre a zona de domicilio urbana e adiposidade corporal excessiva, o que reforça a evidência de que esses são mais vulneráveis, do que as moças, aos fatores ambientais (MALINA, 1990).

Em jovens de ambos os sexos, após controlar para variáveis biológicas e de estilo de vida, o baixo desempenho nos testes de agilidade $e$ potência muscular dos membros inferiores associou-se com excesso de gordura corporal (MOLINDER-URDIALES et al., 2011). Ademais, o desempenho no teste de abdominal explicou, em $10 \%$, a variação do IMC em rapazes (DUMITH et al., 2010). Concordante a isso, no presente estudo, a resistência abdominal inadequada associou-se [RP $=1,22$; IC95\%: 1,14-1,30] com a adiposidade corporal excessiva nos rapazes.

No presente estudo, não foi encontrada associação entre adiposidade corporal excessiva e idade. Concordante a isso, em moças e rapazes (14-18 anos) de Florianópolis, o excesso de adiposidade corporal total, periférica (dobra cutânea do tríceps) e central (dobra cutânea subescapular) não se associou à idade (SILVA et al., 2011). Esse mesmo comportamento foi evidenciado, em adolescentes (14-19 anos) de Pernambuco, ao se utilizar o IMC como indicador de gordura (TASSITANO et al., 2009). Pesquisadores nacionais (DUNCAN et al., 2011) e internacionais (VICENTE-RODRÍGUEZ et al., 2008) que analisaram adolescentes de faixa etária menor e mais ampla, encontraram associação entre idade e excesso de peso. Frente $a$ isso, pode-se entender que, entre as idades de 14 e 17 anos, o acúmulo de gordura corporal se dá de forma equivalente.

O nível econômico mais elevado não apresentou, em nenhum dos sexos, associação com a adiposidade corporal excessiva, o que diverge do observado em Pelotas-RS, em que os maiores níveis econômicos associaram-se, em adolescentes de ambos os sexos, a maior adiposidade corporal (DUQUIA et al., 2008). Tendo em vista que com o aumento da renda as dietas com maior quantidade de açúcar, gordura e densidade energética se tornam preferíveis (DREWNOWSKI, 2000), é possível que a divergência entre os estudos seja decorrente dos níveis de desenvolvimento das localidades. Pelotas-RS apresenta, em relação à JanuariaMG, um maior IDH e renda per capita ( $\underline{\mathrm{ONU}}$, 2000), o que pode favorecer o consumo de alimentos não saudáveis e por consequência, a existência de associação entre nível econômico alto e adiposidade corporal elevada. Já em Januária-MG, o IDH é médio-baixo, o que pode favorecer em menor grau, o consumo de alimentos não saudáveis, não permitindo a observação de associação.

Os índices inadequados de flexibilidade não se associaram com a adiposidade corporal excessiva em nenhum dos sexos, o que é concordante aos achados de Dumith et al. (2010), em que essa valência física não explicou a variação do IMC em escolares e nem apresentou diferenças entre eutróficos, sobrepesados e obesos. Considerando que adolescentes mais e menos ativos apresentam níveis de flexibilidade semelhantes (MELO; OLIVEIRA; ALMEIDA, 2009), pode-se entender que essa valência física não depende da quantidade de atividade física, mas sim de seu padrão (tipo de movimento). Portanto, é possível ter níveis satisfatórios de flexibilidade, com baixo dispêndio energético e por consequência, com níveis excessivos de adiposidade corporal. Apesar disso, a prática de exercícios destinados ao aprimoramento e manutenção da flexibilidade deve ser incentivada, uma vez que, valores satisfatórios para a saúde nessa variável são importantes para prevenir lombalgias e problemas osteoarticulares (HUNT, 2003).

A literatura demonstra que baixos níveis de aptidão aeróbia associam-se com excesso de gordura corporal em adolescentes (MOLINERURDIALES et al., 2011; SILVA et al. 2011), sendo o desempenho cardiorrespiratório a variável da aptidão física que mais explica a variação do IMC (DUMITH et al., 2010). Todavia, no presente estudo, a adiposidade corporal elevada não teve associação com a baixa aptidão aeróbia.

Embora a atividade física moderada e vigorosa relacione-se diretamente com a aptidão aeróbia 
de adolescentes, somente a atividade física intensa relaciona-se inversamente com a gordura corporal (GUTIN et al., 2005). Desse modo, é possível manter níveis satisfatórios de aptidão aeróbia, apenas com a prática de atividade física moderada, sem apresentar valores de gordura corporal adequados. A exemplo disso, no estudo de Glaner (2005a), 88,81\% dos adolescentes atenderam aos critérios de referência para aptidão aeróbia, enquanto $59,55 \%$ atenderam para adiposidade corporal. Assim, a ausência de associação aqui observada pode ser decorrente dos baixos níveis de atividade física intensa, bem como de hábitos alimentares inadequados (GÓMEZ-MARTíNEZ et al., 2012).

Níveis adequados de aptidão cardiorrespiratória reduzem a mortalidade por todas as causas e por doenças cardíacas (KODAMA et al., 2009). Ademais, mesmo sem haver redução na quantidade de gordura corporal, o treinamento aeróbico promove aumento da sensibilidade à insulina e da aptidão cardiorrespiratória (NASSIS et al., 2005). Sendo assim, mesmo que a aptidão aeróbia não tenha apresentado associação com a adiposidade corporal, a prática de exercícios destinados ao aprimoramento e manutenção dessa valência física deve ser incentivada.

Nas moças, nenhuma variável investigada associou-se ao desfecho. Porém, a prevalência de adiposidade corporal elevada foi alta, o que coloca uma maior proporção de moças suscetíveis ao desenvolvimento de obesidade ao atingir a fase adulta (THE et al., 2010). Nessas condições, era esperado que a prevalência de aptidão aeróbia adequada não fosse semelhante entre sexos, mas sim menor entre as moças, haja vista que a aptidão aeróbia se relaciona inversamente com os níveis de adiposidade corporal (MOLINER-URDIALES et al., 2011). Todavia, vale ressaltar que a atividade física intensa, a qual é inversamente relacionada à gordura corporal, é menos presente entre as moças (GUTIN et al., 2005), e que os hábitos alimentares inadequados são mais frequentes entre os adolescentes do sexo feminino (NEUTZLING et al., 2010). Assim, a junção desses dois fatores pode contribuir para que 0 acúmulo excessivo de gordura corporal seja maior nas moças.

Dados do projeto que originou o presente trabalho foram publicados previamente
(PETROSKI et al., 2012; PETROSKI et al., 2011). Em tais trabalhos a composição corporal foi avaliada por meio do IMC (PETROSKI et al., 2012) e utilizando os critérios da Bateria Canadense de aptidão física, que leva em consideração um conjunto de indicadores para avaliação da composição corporal (IMC, circunferência da cintura, dobras cutâneas do tríceps, bíceps, subescapular, supra-ilíaca e panturrilha medial) (PETROSKI et al., 2011). No trabalho de Petroski et al. (2012), não foi encontrado em nenhum dos sexos associação entre o estado nutricional com os componentes da aptidão física (flexibilidade, resistência abdominal e aptidão aeróbia), o que difere do presente estudo que encontrou associação entre adiposidade corporal elevada com resistência abdominal inadequada para os rapazes. No estudo de Petroski et al. (2011) a prevalência de inadequação no componente de composição corporal foi de $25,1 \%$, sendo que a maior proporção de inadequação foi nos rapazes $(32,3 \%)$ em comparação as moças $(18,0 \%)$. Por outro lado, no presente estudo, maior adiposidade corporal elevada foi encontrada no sexo feminino (33,24\%) em comparação ao masculino (13,91\%).

Como limitações, o presente estudo apresenta a impossibilidade de saber se os voluntários se esforçaram ao máximo durante os testes físicos, bem como a não investigação de outros fatores, como cor da pele (DUQUIA et al., 2008), que influencia na adiposidade corporal e poderia alterar as associações observadas na análise ajustada. Outra limitação é o tipo de delineamento que não permite estabelecer uma relação de causa e efeito entre a exposição e o desfecho. Entretanto, é por meio dos estudos de corte transversal que são identificadas associações e levantadas hipóteses, as quais poderão ser mais bem investigadas por estudos prospectivos. A não investigação dos hábitos alimentares, os quais exercem influencia sobre os níveis de adiposidade corporal de adolescentes (GÓMEZMARTîNEZ et al., 2012; DUNCAN et al., 2011) e poderiam alterar as associações observadas na análise ajustada também é outra limitação do estudo. Além disso, outro fator limitante foi a não investigação do nível maturacional, o qual influencia na composição corporal (MALINA, 1991). Porém, vale ressaltar que o intervalo de idade aqui analisado é relativamente breve, e a maior parte da amostra estava na faixa etária de 14-15 anos, o que minimiza a influencia das 
diferenças maturacionais sobre a gordura corporal.

Como pontos fortes do trabalho tem-se uma amostra representativa da população, bem como o uso de técnicas mais acuradas para estimar a gordura corporal, haja vista que dos estudos que investigaram os fatores associados à obesidade, a maioria fez uso do IMC como indicador de gordura corporal (DUNCAN et al., 2011; PELEGRINI et al., 2010; TASSITANO et al., 2009;느 et al., 2008; LIU et al., 2008; $\underline{X U}$ et al., 2008; PELEGRINI et al., 2008), o qual não é um instrumento tão acurado quanto à técnica de dobras cutâneas (REEDMAN et al., 2007). Além disso, o presente estudo traz importantes contribuições, pois relata a prevalência e os fatores associados à adiposidade corporal excessiva. Tais conhecimentos poderão ser utilizados, pelos órgãos governamentais do município, na elaboração e direcionamento das medidas de prevenção e controle do excesso de gordura corporal.

\section{Conclusão}

Pode-se concluir que aproximadamente $25 \%$ dos estudantes de Januária-MG apresentaram adiposidade corporal elevada, sendo a prevalência mais elevada nas moças do que nos rapazes. Assim, uma maior proporção de moças está suscetível ao desenvolvimento de obesidade ao atingir a fase adulta. Além disso, independente da idade, nível econômico, nível de flexibilidade e de aptidão aeróbia, adolescentes do sexo masculino residentes na zona urbana e com baixos níveis de resistência abdominal foram os grupos com maiores probabilidades de excesso de adiposidade corporal. Desse modo, os rapazes parecem ser mais vulneráveis aos fatores ambientais.

\section{Referências}

\begin{abstract}
AAHPERD - AMERICAN ALLIANCE FOR HEALTH, PHYSICAL EDUCATION, RECREATION AND DANCE: Physical best. Reston: 1988.
\end{abstract}

\section{ABEP - ASSOCIAÇÃO BRASILEIRA DE} EMPRESAS DE PESQUISA. Critério de Classificação Econômica Brasil, São Paulo, SP, 2008. Disponível em:

$<$ http://www.abep.org/codigosguias/Criterio Brasil 2008.pdf>. Acesso em: 26 ago. 2008.

DANIELS, S. R. The use of BMI in the clinical setting. Pediatrics, Springfield, v. 124, n.S1 p.
S35-41, 2009. Disponível em:

<http://pediatrics.aappublications.org/content/124/ Supplement 1/S35.full.pdf+html>. Acesso em: 10/08/2012.

DELLAGRANA, R. A.; SILVA, M. P.; SMOLAREK, A. C.; BOZZA, R.; STABELINI NETO, A.; CAMPOS, W. Composição corporal, maturação sexual e desempenho motor de jovens praticantes de handebol. Motriz, Rio Claro, v.16 n.4 p.880888, 2010. Disponível em:

$<$ http://www.periodicos.rc.biblioteca.unesp.br/inde x.php/motriz/article/view/1980-

6574.2010v16n4p880/pdf 2 >. Acesso em: $07 / 04 / 2011$.

DREWNOWSKI, A. Nutrition transition and global dietary trends. Nutrition, Burbank, v. 16, n. 7-8, p. 486-7, 2000. Disponível em:

$<$ http://www.nutritionjrnl.com/article/S08999007(00)00295-1/abstract>. Acesso em: $25 / 06 / 2011$.

DUMITH, S. C.; RAMIRES, V. V.; SOUZA, M. A.; MORAES, D. S.; PETRY, F. G.; OLIVEIRA, E. S. et al. Overweight/obesity and physical fitness among children and adolescents. Journal of Physical Activity and Health, Champaign, v. 7, n. 5, p. 641-648, 2010

DUNCAN, S.; DUNCAN, E. K.; FERNANDES, R. A.; BUONANI, C.; BASTOS, K. D.; SEGATTO, A. $F$. et al. Modifiable risk factors for overweight and obesity in children and adolescents from São

Paulo, Brazil. BMC Public Health, London, v. 22, n. 11, p. 585, 2011. Disponível em: $<$ http://www.ncbi.nlm.nih.gov/pmc/articles/PMC31 54175/pdf/1471-2458-11-585.pdf >. Acesso em: 10/08/2012.

DUQUIA, R. P.; DUMITH, S. C.; REICHERT, F. F.; MADRUGA, S. W.; DURO, L. N.; MENEZES, A. M. B. et al. Epidemiologia das pregas cutâneas triciptal e subescapular elevadas em adolescentes. Caderno de Saúde Pública, Rio de Janeiro, v. 24, n.1, p. 113-121, jan, 2008. Disponível em: $<$ http://www.scielo.br/pdf/csp/v24n1/10.pdf >. Acesso em: 25/03/2011.

FREEDMAN, D. S.; WANG, J.; OGDEN, C. L.; THORNTON, J. C.; MEI, Z.; PIERSON, R. N. et al. The prediction of body fatness by BMI and skinfold thicknesses among children and adolescents. Annals of Human Biology, London, v. 34, n. 2, p. 183-94, 2007.

GLANER, M. F. Aptidão física relacionada à saúde de adolescentes rurais e urbanos em relação a critérios de referência. Revista Brasileira de Educação Física e Esporte, São Paulo, v.19, n.1, p.13-24, 2005a. Disponível em: 
$<$ http://www.revistasusp.sibi.usp.br/pdf/rbefe/v19n 1/v19n1a02.pdf >. Acesso em: 10/04/11.

GLANER, M. F. Índice de massa corporal como indicativo da gordura corporal comparado às dobras cutâneas. Revista Brasileira de Medicina do Esporte, Niterói, v.11, n.4, p. 243-46, 2005b. Disponível

em:<http://www.scielo.br/pdf/rbme/v11n4/26867.p df $>$. Acesso em: 10/04/11.

GÓMEZ-MARTÍNEZ, S.; MARTÍNEZ-GÓMEZ, D.; PEREZ DE HEREDIA, F.; ROMEO, J.; CUENCAGARCIA, M.; MARTÍN-MATILLAS, M, et al. Eating habits and total and abdominal fat in Spanish adolescents: influence of physical activity. The AVENA study. The Journal of Adolescent Health, New York, v. 50, n. 4, p.403-9, 2012.

GORDIA, A. P.; QUADROS, T. M. B.; CAMPOS, W.; PETROSKI, E. L . Nível de atividade física em adolescentes e sua associação com variáveis sociodemográficas. Revista Portuguesa de Ciências do Desporto, Porto, v. 10, n.1, p. 172-9, 2010. Disponível em:<

http://www.scielo.gpeari.mctes.pt/pdf/rpcd/v10n1/v 10n1a06.pdf>. Acesso em: 10/08/2012.

GUTIN, B.; YIN, Z.; HUMPHRIES, M. C.; BARBEAU, P. 1.Relations of moderate and vigorous physical activity to fitness and fatness in adolescents. The American journal of clinical nutrition, Bethesda, v. 81, n. 4, p. 746-750, 2005. Disponível em: <

http://ajcn.nutrition.org/content/81/4/746.full.pdf+ht $\underline{\mathrm{ml}}$ >. Acesso em: 10/08/2012.

HUNT, A. Musculoskeletal fitness: the keystone in overall well-being and injury prevention. Clinical Orthopaedics and Related Research, Philadelphia, v. 409, p. 96-105, 2003.

IBGE - INSTITUTO BRASILEIRO DE GEOGRAFIA E ESTATÍSTICA. IBGE cidades, 2010. Disponível em:

$<$ http://www.ibge.gov.br/cidadesat/topwindow.htm ?1>. Acesso em: 20/01/2012.

KODAMA, S.; SAITO, K.; TANAKA, S.; MAKI, M.; YACHI, Y.; ASUMI, M. et al. Cardiorespiratory fitness as a quantitative predictor of all-cause mortality and cardiovascular events in healthy men and women: a meta-analysis. The Journal of the American Medical Association, Chicago, v. 301, n. 19, p. 2024-35, 2009. Disponível em:

$<$ http://jama.amaassn.org/content/301/19/2024.full.pdf+html>. Acesso em: 25/07/2011.

LI, M.; DIBLEY, M. J.; SIBBRITT, D.; YAN, H. Factors associated with adolescents' overweight and obesity at community, school and household levels in Xi'an City, China: results of hierarchical analysis. European Journal of Clinical

Nutrition, London, v. 62, n. 5, p. 635-43, 2008.

LIU, J.; BENNETT, K. J.; HARUN, N.; PROBST, J. $C$. Urban-rural differences in overweight status and physical inactivity among US children aged 10-17 years. The Australian Journal of Rural Health, Armidale, v. 24, n. 4, p. 407-15, 2008. Disponível em:

$<$ http://onlinelibrary.wiley.com/doi/10.1111/j.1748$0361.2008 .00188 . \mathrm{x} / \mathrm{pdf}>$. Acesso em: $25 / 07 / 2011$.

\section{LOHMAN, T.G. Advances in body} composition assessment. Champaign(II): Human Kinetics, 1992.

MALDONADO, G.; GREENLAND, S. Simulation study of confounder-selection strategies.

American Journal of Epidemiology, Baltimore, v. 138 , n. 1, p. 923-936, 1993. Disponível em: $<$ http://ije.oxfordjournals.org/content/26/1/224.full. pdf+html >. Acesso em: 10/03/2010.

MALINA, R. M. Crescimento de crianças latino americanas: comparação entre os aspectos sócio-econômicos, urbano-rural, e tendência secular. Revista Brasileira de Ciência e

Movimento, São Caetano do Sul, v. 4, n. 3, p. 4675, 1990. Disponível em:

$<$ http://portalrevistas.ucb.br/index.php/RBCM/articl e/view/145/321>. Acesso em: 27/08/2011.

MALINA, R. M. Growth, maturation, and physical activity. Champaign: Human Kinetics Book, 1991.

MELO, F. A. P.; OLIVEIRA, F. M. F; ALMEIDA, M. $B$. Nível de atividade física não identifica o nível de flexibilidade de adolescentes. Revista Brasileira de Atividade Física e Saúde, Pelotas, v. 14, n.1, p. 48-54, 2009. Disponível em: $<$ http://www.sbafs.org.br/ artigos/92.pdf>. Acesso em: 10/08/2012.

MOLINER-URDIALES, D.; RUIZ, J. R.; VICENTERODRIGUEZ, G.; ORTEGA, F. B.; REY-LOPEZ, J. P.; ESPAÑA-ROMERO, V.; et al. Associations of muscular and cardiorespiratory fitness with total and central body fat in adolescents: The HELENA Study. British Journal of Sports Medicine, London, v. 45, n. 2, p. 101-108, 2011. Disponível em: <http://www.helenastudy.com/files/MolinerUrdiales-BJM-2011.pdf>. Acesso em: 10/07/2011.

NASSIS, G. P.; PAPANTAKOU, K.; SKENDERI, K.; TRIANDAFILLOPOULOU, M.; KAVOURAS, S. A.; YANNAKOULIA, M. et al. Aerobic exercise training improves insulin sensitivity without changes in body weight, body fat, adiponectin, and inflammatory markers in overweight and obese girls. Metabolism: clinical and experimental, New York, v. 54, n. 11, p. 1472-9, 2005. 
NEUTZLING, M. B.; ASSUNÇÃO, M. C. F.; MALCON, M. C.; HALLAL, P. C.; MENEZES, A. M. B. Hábitos alimentares de escolares adolescentes de Pelotas, Brasil. Revista de Nutrição, Campinas, v. 23, n. 3, p. 379-388, 2010. Disponível em:<http://dx.doi.org/10.1590/S141552732010000300006 >. Acesso em: 25/08/11.

ONU - ORGANIZAÇÃO DAS NAÇÕES UNIDAS. Ranking do IDH-M dos municípios do Brasil PNUD Brasil, 2000.

ORTEGA, F. B.; RUIZ, J. R.; CASTILLO, M. J.; SJÖSTRÖM, M. Physical fitness in childhood and adolescence: a powerful marker of health.

International Journal of Obesity, England, v. 32, n. 1, p.1-11, 2008. Disponível em:

$<$ http://www.nature.com/ijo/journal/v32/n1/full/0803 774a.html>. Acesso em: 10/08/2012.

PEEBLES, R. Adolescent obesity: etiology, office evaluation, and treatment. Adolescent medicine: state of the art reviews, Elk Grove Village, v. 19, n. 3, p. 380-405, 2008. Disponivel em: < http://peds.stanford.edu/Rotations/pec ccp/docum ents/ObesityArticle1.pdf>. Acesso em: 15/08/2012.

PELEGRINI, A.; SILVA, D. A. S.; PETROSKI, E. L.; GLANER, M. F. Estado nutricional e fatores associados em escolares domiciliados na área rural e urbana. Revista de Nutrição, Campinas, v. 23, n. 5, p. 839-46, 2010.

$<$ http://dx.doi.org/10.1590/S141552732010000500014>._Acesso em: 20/08/2011.

PETROSKI, E. L. (Org.). Antropometria: técnicas e padronizações. $4^{a}$ edição. Porto Alegre: Editora Palloti, 2009.

PETROSKI, E.L.; SILVA, A.F.; RODRIGUES, A.B.; PELEGRINI, A. Associação entre baixos níveis de aptidão física e fatores sóciodemográficos em adolescentes de área urbanas e rurais. Revista Motricidade, Santa Maria da Feira, v. 8, n. 1, p. 5-13, 2012.<http://dx.doi.org/10.6063/motricidade.8(1).2 $\underline{27}$ >. Acesso em: 20/08/2012.

PETROSKI, E.L.; SILVA, A.F.; RODRIGUES, A.B.; PELEGRINI, A. Aptidão física relacionada à saúde em adolescentes brasileiros residentes em áreas de médio/baixo índice de desenvolvimento humano. Revista de Salud Pública, Bogotá, v. 13, n. 2, p. 219-228, 2011. $<$ http://dx.doi.org/10.1590/S012400642011000200004>. Acesso em: 20/08/2012.

SHI, Z.; HOLMBOE-OTTESEN, G. Sociodemographic differences in food habits and preferences of school adolescents in Jiangsu Province, China. European Journal of Clinical
Nutrition, London, v. 59, n. 12, p. 1439-1448, 2005. Disponível em: $<$ http://www.nature.com/ejcn/journal/v59/n12/pdf/1 602259a.pdf>. Acesso em 17/08/2011.

SILVA, D. A. S.; PELEGRINI, A.; SILVA J. M. F. L.; PETROSKI, E. L. Epidemiology of whole body, peripheral, and central adiposity in adolescents from a Brazilian state capital. European Journal of Pediatrics, Berlim, in press, 2011.

SILVA, M. C.; PACCINI, M. K.; GLANER, M. F. Aptidão física relacionada à saúde de adolescentes oriundos de diferentes níveis econômicos. Revista da Educação Física, Maringá, v. 18, n. 2, p. 199-206, 2007. Disponível em:

$<$ http://periodicos.uem.br/ojs/index.php/RevEducFi s/article/view/3276/2342>. Acesso em: 20/08/2011.

TASSITANO, R. M.; BARROS, M. V. G.; TENÓRIO, M. C. M.; BEZERRA, J.; HALLAL, P. C. Prevalência e fatores associados ao sobrepeso e à obesidade em adolescentes, estudantes de escolas de Ensino Médio de Pernambuco, Brasil. Caderno de Saúde Pública, Rio de Janeiro, v. 25, n. 12, p. 2639-2652, 2009. $<$ http://dx.doi.org/10.1590/S0102311X2009001200010>. Acesso em: 20/08/2011.

THE, N. S.; SUCHINDRAN, C.; NORTH, K. E.; POPKIN, B. M. GORDON-LARSEN, P. Association of adolescent obesity with risk of severe obesity in adulthood. The Journal of the American Medical Association, Chicago, v. 304, n.18, p.2042-7, 2010. Disponivel em: $<$ http://jama.amaassn.org/content/304/18/2042.full.pdf+html>. Acesso em: 12/08/2011.

VICENTE-RODRÍGUEZ, G.; REY-LÓPEZ, J. P.; MARTÍN-MATILLAS, M.; MORENO, L. A.; WÄRNBERG, J.; REDONDO, C.; TERCEDOR, $P$. et al. Television watching, videogames, and excess of body fat in Spanish adolescents: The AVENA study. Nutrition, Burbank, v. 24, n. 7-8, p. 654-62, 2008..

VICTORA, C. G.; HUTTLY, S. R.; FUCHS, S. C.; OLINTO, M. T. The role of conceptual frameworks in epidemiological analisys: a hierarchical approach. International Journal of Epidemiology, London, v. 26, n.1, p. 224-227, 1997. Disponível em: $<$ http://ije.oxfordjournals.org/content/26/1/224.full. pdf+html >. Acesso em: 10/03/2010.

XU, F.; LI, J.; WARE, R. S.; OWEN, N.

Associations of television viewing time with excess body weight among urban and rural high-school students in regional mainland China. Public 
Health Nutrition, Wallingford, n 11, n. 9, p. 891-6, 2008.

\section{Endereço:}

Diego Augusto Santos Silva UFSC/CDS - Núcleo de Cineantropometria e Desempeno Humano

Campus Universitário - Trindade

Florianópolis SC Brasil

88040-900 Caixa Postal 476

Telefone/Fax: (48) 37218562

e-mail:diegoaugustoss@yahoo.com.br

Recebido em: 7 de setembro de 2011.

Aceito em: 27 de janeiro de 2013.

\section{(c) $(7)$}

Motriz. Revista de Educação Física. UNESP, Rio Claro, SP, Brasil - elSSN: 1980-6574 - está licenciada sob Creative Commons - Atribuicão 3.0 